\title{
A87 INVOLVEMENT OF TOLL-LIKE RECEPTOR 2 IN PRISTANE-INDUCED LUPUS
}

Vilma Urbonaviciute, ${ }^{1}$ Charlotte Starke, ${ }^{1}$ Daniela Graef, ${ }^{1}$ Markus Mroz, ${ }^{2}$ Carsten Kirschning, ${ }^{3}$ Georg Schett, ${ }^{2}$ Reinhard E Voll ${ }^{1,4}{ }^{1}$ Nikolaus Fiebiger Center of Molecular Medicine, University Hospital Erlangen, Erlangen, Germany; ${ }^{2}$ Department Internal Medicine 3, University Hospital Erlangen, University of Erlangen-Nuremberg, Erlangen, Germany; ${ }^{3}$ Institute of Medical Microbiology, University of Duisburg-Essen, Duisburg, Germany; ${ }^{4}$ Department of Rheumatology and Clinical Immunology, University Hospital Freiburg, Freiburg, Germany

10.1136/ard.2010.148973.5

Background Autoantibodies against double stranded (ds) DNA and nucleosomes represent a hallmark of systemic lupus erythematosus (SLE). ${ }^{1}$ However, the mechanisms involved in breaking the immunological tolerance against these poorly immunogenic nuclear components are not fully understood. Recent data indicate that toll-like receptors (TLRs) recognising endogenous ligands may be critically involved in the breaking of peripheral tolerance against nuclear autoantigens. ${ }^{2}$ Results of this recent studies in non-autoimmune mice provide evidence of a important role of TLR2 in the anti-dsDNA and antihistone $\operatorname{Ig} G$ autoantibody induction by high mobility group box protein 1-nucleosome complexes derived from apoptotic cells. ${ }^{3}$ Objective Using the pristane-induced mouse model of SLE, the authors further investigated the requirement of TLR2 signalling for induction of lupus-specific autoantibody production and SLE like disease.

Methods Female C57BL/6 wild-type (WT), TLR2-deficient mice were injected intraperitoneally with a single dose of 500 $\mu 1$ of the hydrocarbon oil pristane. The numbers of plasma cells were determined by flow cytometry. The concentrations of autoantibodies in sera were measured by ELISA Autoantibody-secreting cells in the kidneys were detected by enzyme-linked immunosorbent spot assay. Renal disease was assessed by semiquantitative measurement of proteinuria in spot urine and quantified over $24 \mathrm{~h}$ after collection in metabolic cages as well as by histological analyses.

Results TLR2 ${ }^{-/}$mice generated reduced numbers of splenic CD138/cytoplasmic $\kappa / \lambda$-L chain ${ }^{+} / C D 25$ plasma cells in response to pristane treatment. Anti-dsDNA, antihistone, antinucleosome and some antinuclear antibody $\operatorname{Ig} G$ responses were delayed and significantly reduced in pristane-treatedTLR2 ${ }^{-/}$mice compared to pristane-treated WT controls. There were markedly lower numbers of total IgG-secreting and anti-dsDNA specific IgG-secreting cells in the kidneys of pristane-treated-TLR $2^{-/-}$mice in comparison to C57BL/6 mice. Importantly, pristane-treated TLR2-deficient mice developed significantly milder renal disease compared to the WT control group.

Conclusion TLR2 is specifically required for the lupus-specific autoantibody production as well as for development of renal disease in pristane-induced murine lupus model. Specific blocking of TLR2 signalling may therefore be a promising novel strategy for treatment of SLE. 


\section{REFERENCES}

Hahn BH. Antibodies to DNA. N Engl J Med 1998;338:1359-68.

2. Marshak-Rothstein A. Toll-like receptors in systemic autoimmune disease. Nat Rev Immunol 2006; 6:823-35.

3. Urbonaviciute V, Fürnrohr BG, Meister S, et al. Induction of inflammatory and immune responses by HMGB1-nucleosome complexes: implications for the pathogenesis of SLE. J Exp Med 2008;205:3007-18. 